\title{
Influence of divalent cations in the protein crystallization process assisted by Lanthanide-based additives.
}

Amandine Roux, ${ }^{[a, b]}$ Romain Talon, ${ }^{[c]}$ Zaynab Alsalman, ${ }^{[c]}$ Sylvain Engilberge, ${ }^{[c]}$ Anthony D’Aléo, [a] Sebastiano Di Pietro, ${ }^{[a]}$ Adeline Y. Robin, ${ }^{[c]}$ Alessio Bartocci, [a] Guillaume Pilet, ${ }^{[d]}$ Elise Dumont, ${ }^{[a, e]}$ Tristan Wagner, ${ }^{[f, g]}$ Seigo Shima, ${ }^{[f]}$ François Riobé, ${ }^{[a] *}$ Eric Girard, ${ }^{[c] *}$ Olivier Maury. ${ }^{[a]^{*}}$

a] Univ Lyon, ENS de Lyon, CNRS UMR 5182, Université Claude Bernard Lyon 1, Laboratoire de Chimie, F-69342 Lyon, France.

E-mail: olivier.maury@ens-lyon.fr, francois.riobe@ens-lyon.fr,

[b] Polyvalan F-69342 Lyon, France

[c] Univ Grenoble Alpes, CEA, CNRS, IBS, F-38000 Grenoble, France.

E-mail: eric.girard@ibs.fr.

[d] Univ de Lyon, CNRS UMR 5615, Université Claude Bernard Lyon 1, 43 boulevard du 11 novembre 1918, F-69622 Villeurbanne cedex.

[e] Institut Universitaire de France, 1 rue Descartes, 75005 Paris

[f] Microbial Protein Structure Group, Max Planck Institute for Terrestrial Microbiology, Karl-von-Frisch-Str. 10, D-35043 Marburg, Germany

[g] Microbial Metabolism Group, Max Planck Institute for Marine Microbiology, 1-Celsiusstrasse, 35043 Bremen, Germany 


\section{1) Lanthanide complex syntheses}

Starting reagents were purchased from Sigma-Aldrich or Acros and used without further purification. All organic solvents were dried on molecular sieves for $48 \mathrm{~h}$.

Synthesis of the complexes $[\mathrm{Na}]_{3}\left[\mathrm{Eu}(\mathrm{DPA})_{3}\right]^{1}$, and $\mathrm{Tb}-\mathrm{Xo}^{2,3}$ have been previously described Both complexes are now commercially available by Polyvalan company.

\section{2) Protein production and crystallization}

The Ec-AcrB (Acriflavine resistant protein B from Escherichia coli) overexpression vector is a gift of K.M. Pos. Ec-AcrB production and purification were performed as described in the literature. ${ }^{4}$ Prior to crystallization assays, Ec-AcrB was concentrated to $10 \mathrm{mg} \cdot \mathrm{ml}^{-1}$ in $10 \mathrm{mM}$ HEPES pH 7.0, 0.02\% n-dodecyl- $\beta$-D-maltopyranoside (DDM). Crystallization of Ec-AcrB in the presence and in the absence of $[\mathrm{Na}]_{3}\left[\mathrm{Eu}(\mathrm{DPA})_{3}\right]$ was performed according to the protocol used for the self-crystallization evaluation of $[\mathrm{Na}]_{3}\left[\mathrm{Eu}(\mathrm{DPA})_{3}\right]$.

Production of adenylate kinase (AdkA) protein from Methanothermococcus thermolithotrophicus as well as crystallization in the presence of $\mathrm{Tb}$-Xo4 was performed as indicated in the literature. ${ }^{5}$

\section{3) Computational details.}

In order to estimate the interaction energies for Tb-Xo4 at AdkA binding site, we started from the PDB structure obtained for AdkA co-crystallized with Tb-Xo4 (PDB ID 6HF7), and resort to density functional theory (DFT) calculations to situate the energetic contributions of each residues or molecule involved in the binding site of AdkA. The computational protocol was identical to the one used in a previous publication ${ }^{6}$ for 6 proteins co-crystallized with Tb-Xo4, namely after addition of relevant hydrogens based on a PropKa analysis ${ }^{7}$ and corroborated by chemical inspection, a geometry optimization of the cluster embracing the Tb-Xo4 complex, the glycerol molecule, the aspartate $\mathrm{D} 90_{\mathrm{C}}$, with the bridging magnesium ion and its five coordinated water molecules, all present in the X-ray structure was performed at the B3LYPD3BJ/6-31G(d,p) level of theory. The Stuttgart/Dresden SDD pseudo-potential was used for the terbium atom. The calculations were performed with the Gaussian16 Rev B.01 series of programs. ${ }^{8}$ We then extracted interaction energies based on the cluster represented in Figure S7, which reflect the contribution of each residue in a static framework at $0 \mathrm{~K}$. An implicit continuum model was used (IEFPCM as available in Gaussian16) with an effective dielectric constant of 8.0 to account for the electrostatic embedding of the protein. 


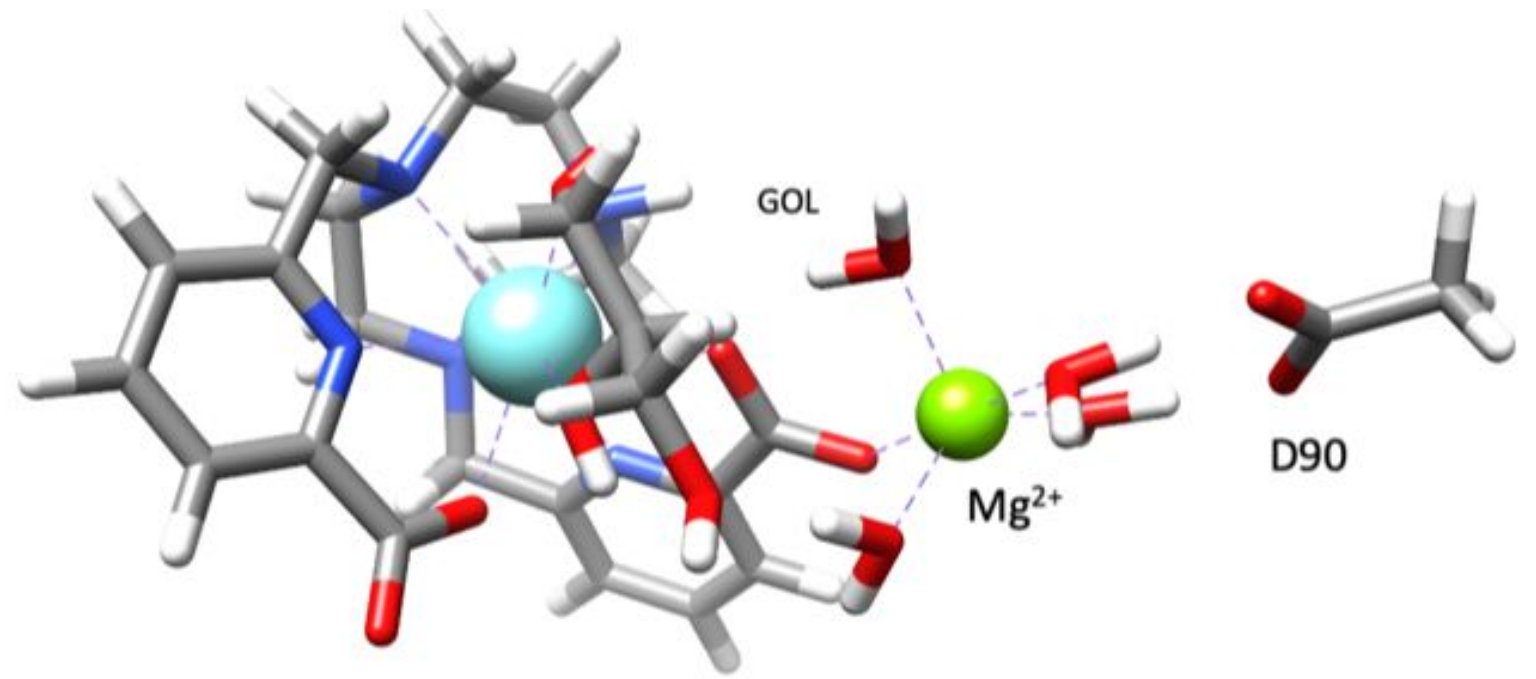

Figure S1. Cluster of $\mathrm{Tb}-\mathrm{Xo} 4$, interacting with a glycerol molecule, as well as $\mathrm{Mg}^{2+}$ and the aspartate D90. The four water molecules were taken from the crystallographic positions of their oxygen atoms. Residue D90 was capped by a methyl group, and the C $\square$ atoms were maintained in their crystallographic position.

Table S1. Interaction energies estimated at the DFT/B3LYP-6-31+G(d,p)/SDD level of theory, as well as characteristic distances. $\left(^{*}\right)$ The distances between oxygen atoms of the four water molecules and the central magnesium atom range between 1.99 and $2.09 \AA$.

\begin{tabular}{|l|c|c|}
\hline Residues & Distances $(\AA)$ & Interaction energies $\left(\mathrm{kcal}^{\mathrm{m}} \mathrm{mol}^{-1}\right)$ \\
\hline Glycerol & $2.51,2.51$ & -27.8 \\
\hline $\mathrm{D} 90$ & $1.52,1.53$ & -9.3 \\
\hline$\left\{\mathrm{Mg}^{2+}+4 \mathrm{H}_{2} \mathrm{O}+\mathrm{D} 90\right\}$ & $2.09\left(^{*}\right)$ & -25.3 \\
\hline & & \\
\hline
\end{tabular}

\section{4) Self-crystallization evaluation}

Prior to automated crystallization experiments, the lanthanide complex powder was directly dissolved in milliQ water to reach the required concentration $(25 \mathrm{mM}$ and $100 \mathrm{mM}$ for $[\mathrm{Na}]_{3}\left[\mathrm{Eu}(\mathrm{DPA})_{3}\right]$ and $10 \mathrm{mM}$ and $100 \mathrm{mM}$ for Tb-Xo4).

All automated screening trials were done at the High-Throughput Crystallization Laboratory (HTXlab) platform (EMBL Grenoble). The crystallization experiments were set up by mixing a volume of lanthanide complex solution with an equal volume of precipitant solution to form the crystallization drops. Storage (at 293K) and crystallization drops inspection were ensured automatically by the imaging system available at the HTXlab. We selected standard commercial crystallization kits as proposed by the HTXlab for initial screening, for a total of 576 conditions.

The kits used for self-crystallization evaluation of $\mathrm{Tb}-\mathrm{Xo} 4$ and $[\mathrm{Na}]_{3}\left[\mathrm{Eu}(\mathrm{DPA})_{3}\right]$ are detailed in Tables S1 and S2. 
Table S2. Commercial crystallization kits used with $[\mathrm{Na}]_{3}\left[\mathrm{Eu}(\mathrm{DPA})_{3}\right]$ ( ${ }^{*} \mathrm{Kits}$ containing divalent ions)

\begin{tabular}{|c|c|c|}
\hline Screen 1 & Hampton Research & Crystal Screen I \& II* \\
\hline Screen 2 & Hampton Research & Crystal Screen lite \& PEG/Ion* \\
\hline Screen 3 & Qiagen & MembFac \& Natrix* \\
\hline Screen 4 & Qiagen & $\begin{array}{l}\text { Quick Screen \& grid screens sulfate, formate, } \\
\text { malonate }\end{array}$ \\
\hline Screen 5 & Hampton Research & Grid Screen PEG 6K, PEG/LiCl, MPD - Screen Mme \\
\hline Screen 6 & Hampton Research & Index Screen* \\
\hline
\end{tabular}

Table S3. Commercial crystallization kits used with Tb-Xo4 ("Kits containing divalent ions)

\begin{tabular}{lll} 
Screen 1 & Qiagen/Nextal & The Classics Suite* \\
\hline Screen $\mathbf{2}$ & Molecular dimensions & JCSG+* $^{*}$ \\
\hline Screen 3 & Molecular dimensions & PACT premier* \\
\hline Screen 4 & Qiagen/Nextal & PEGs-l* \\
\hline Screen 5 & Hampton Research & Grid Screen Salt \\
\hline Screen 6 & Rigaku Reagents & Wizard I \& II \\
\hline
\end{tabular}

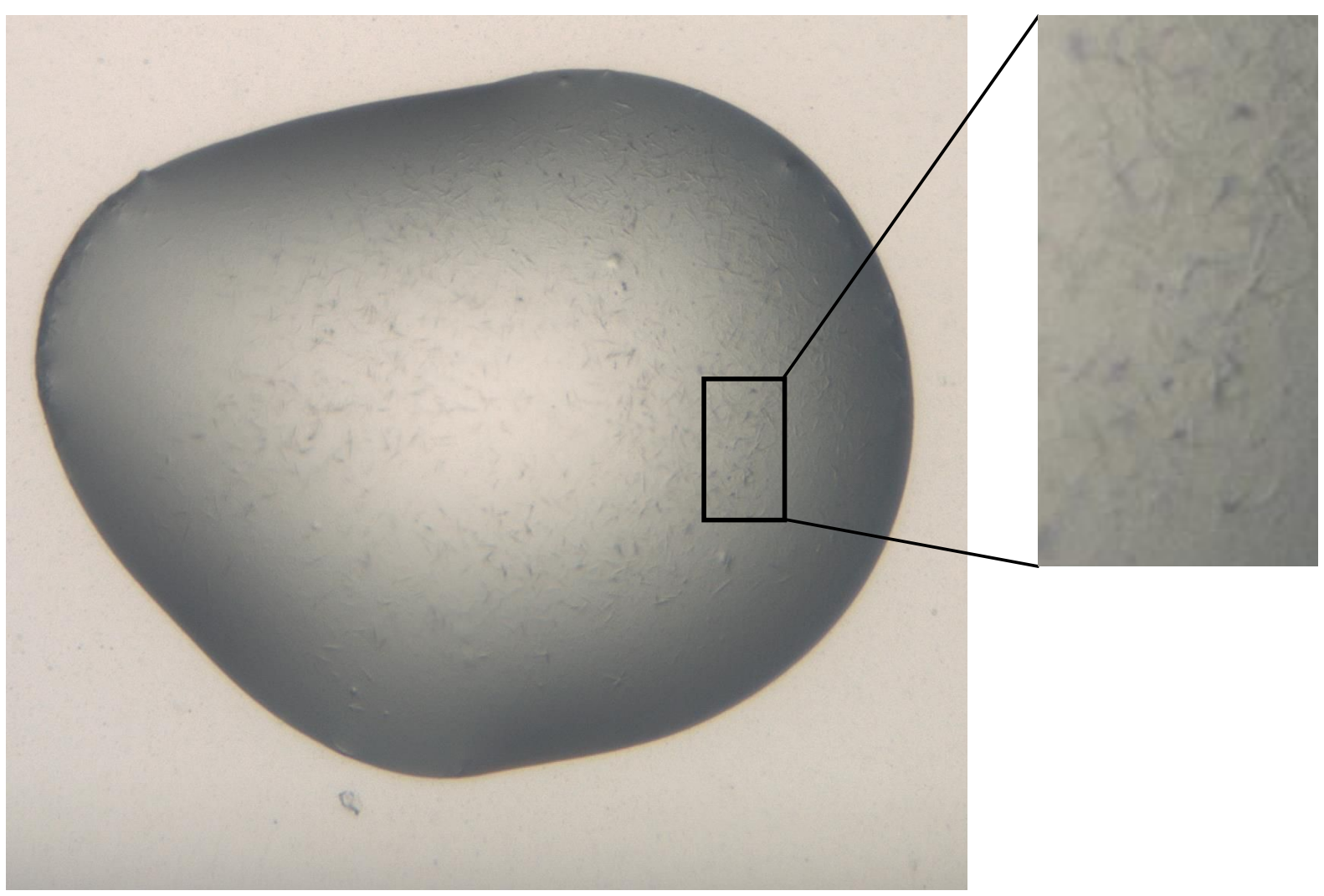

Figure S2. Self-crystallization or precipitation of $\mathrm{Tb}-\mathrm{Xo}_{\mathrm{O}}$ (at $0.1 \mathrm{M}$ concentration) during automated HTX experiments in the following condition: crystallization kit PEGs-l; condition E3; composition: ammonium fluoride 0.2M, PEG 3350 20\%. 


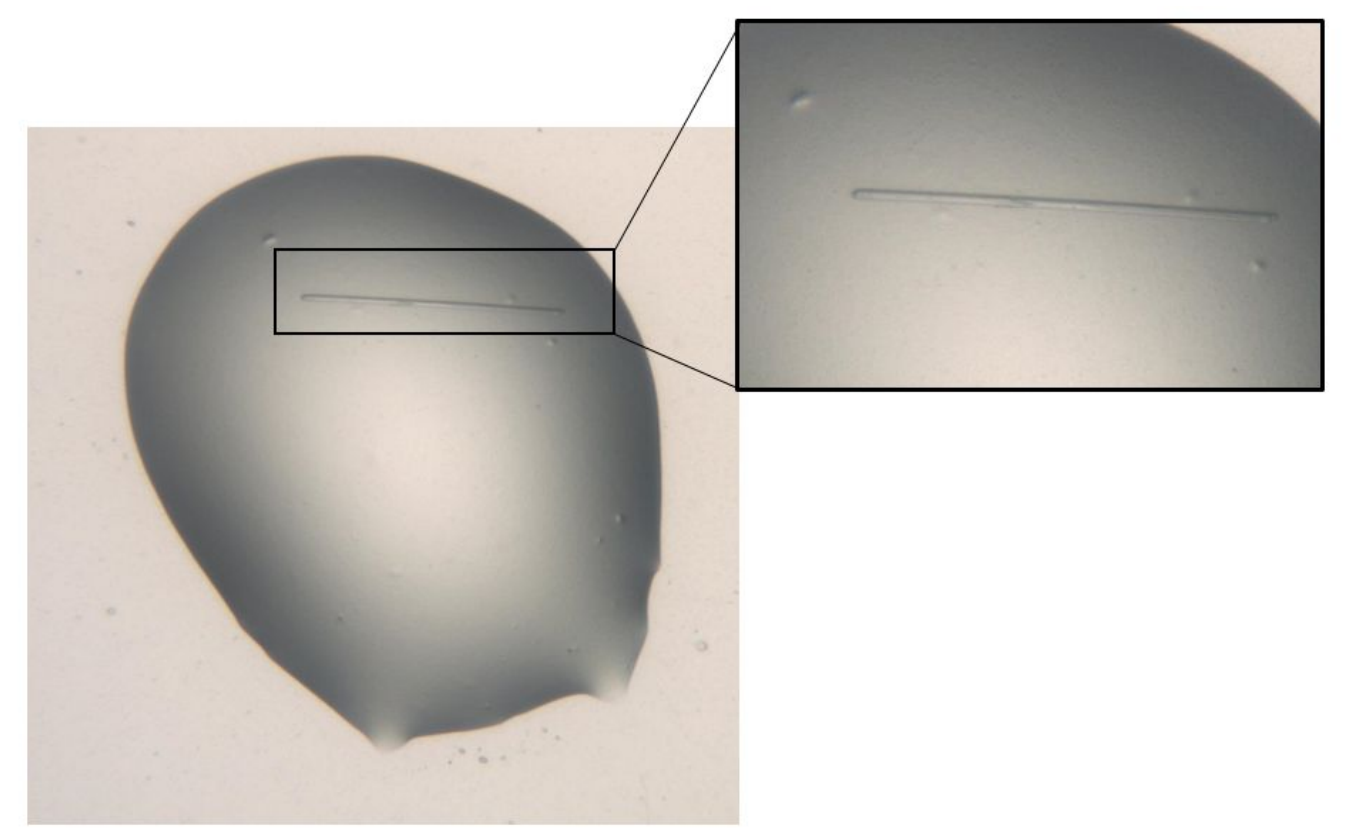

Figure S3. Self-crystallization of $\mathrm{Tb}-\mathrm{Xo}_{\mathrm{O}}$ (at $0.1 \mathrm{M}$ concentration) during automated HTX experiments in the following condition: crystallization kit JCSG; condition D10; composition: calcium acetate hydrate $0.2 \mathrm{M}$, sodium cacodylate $0.1 \mathrm{M}$, pH 6.5, PEG $30040 \%$.

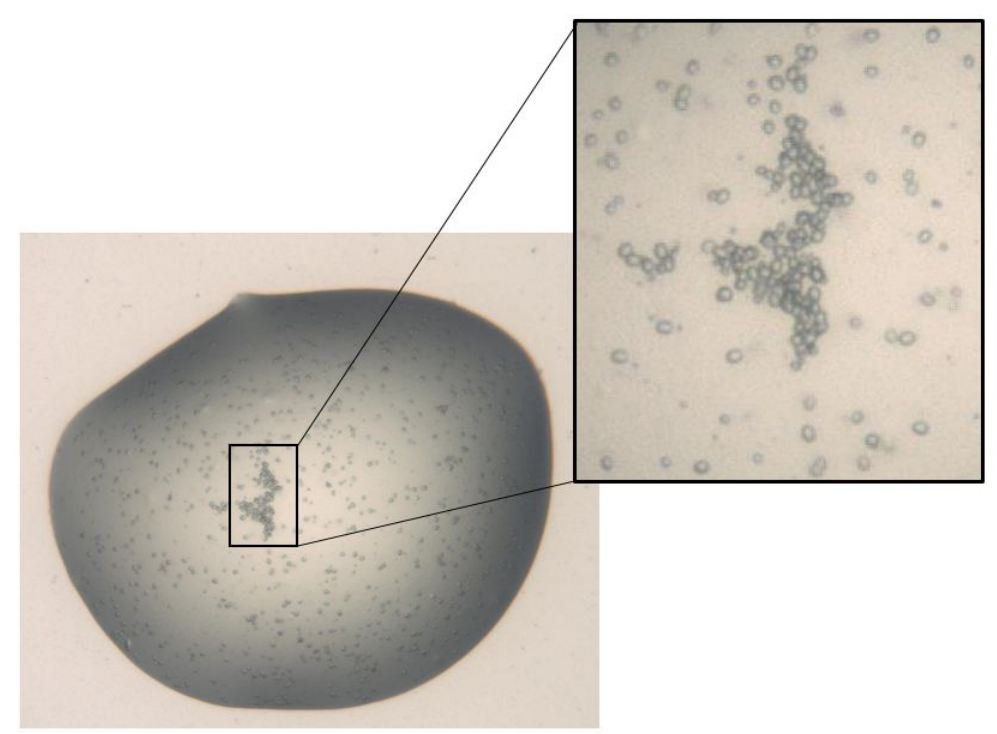

Figure S4. Self-crystallization of Tb-Xo4 (at $0.1 \mathrm{M}$ concentration) during automated HTX experiments in the following condition: crystallization kit The Classics suite; condition F10; composition: ammonium sulfate $0.2 \mathrm{M}$, sodium cacodylate $0.1 \mathrm{M}, \mathrm{pH} 6.5$, PEG $800030 \%$.

\section{5) Single-Crystal X-ray diffraction}

Good quality single-crystals of 1, 2, 3 and 4 were mounted on an Oxford Diffraction Gemini equipped with graphite monochromatized Mo Ka radiation $(\lambda=0.71073 \AA)$ fine-focus sealed tubes. Intensity data were collected at 293. Crystal data were collected using a Gemini (Oxford Diffraction, 2006). ${ }^{9}$ Data refinement and reduction were performed using the CrysAlis 
software. Analytical absorption corrections were applied to the intensity values. ${ }^{10,11}$ The structures were solved by direct methods using the programs SIR $97^{12}$ and refined using the programs CRYSTALS. ${ }^{13}$ All non-hydrogen atoms were refined anisotropically. C-bound hydrogen atoms in 1 were placed geometrically and refined using a riding model approximation. $\mathrm{H}$ atoms for water (coordinated or not) were located in the difference map and the positions were restrained to minimize $\mathrm{H} \cdots \mathrm{H}$ clashes. In case of complex 1 , we present the best model we have been able to obtain concerning potential $\mathrm{H} \cdots \mathrm{H}$ clashed.

CCDC-1405178 (1), -2109355 (2), -1405177 (3) and -2041987 (4) references contain the supplementary crystallographic data. These data can be obtained free of charge from The Cambridge Crystallographic Data Centre via www.ccdc.cam.ac.uk/data request/cif 
Table S4. Crystallographic details and structure refinement results for complexes 1, 2, 3 and 4.

\begin{tabular}{|c|c|c|c|c|}
\hline & 1 & 2 & 3 & 4 \\
\hline Formula & $\mathrm{C}_{42} \mathrm{H}_{66} \mathrm{Eu}_{2} \mathrm{Mg}_{3} \mathrm{~N}_{6} \mathrm{O}_{49}$ & $\mathrm{C}_{21} \mathrm{H}_{31} \mathrm{Ca}_{1.50} \mathrm{Eu}_{1} \mathrm{~N}_{3} \mathrm{O}_{23}$ & $\mathrm{C}_{7} \mathrm{H}_{7} \mathrm{Cu}_{1} \mathrm{~N}_{1} \mathrm{O}_{6}$ & $\mathrm{C}_{20} \mathrm{H}_{26.50} \mathrm{Cl}_{2} \mathrm{~N}_{5} \mathrm{O}_{7} \mathrm{~Tb}_{1}$ \\
\hline Formula weight $\left(\mathrm{g} \cdot \mathrm{mol}^{-1}\right)$ & 1815.8 & 905.56 & 264.7 & 678.8 \\
\hline Crystal system & monoclinic & monoclinic & monoclinic & tetragonal \\
\hline Space group & $\mathrm{Pc}$ & $\mathrm{P} 2 / \mathrm{c}$ & $\mathrm{P} 2_{1} / \mathrm{c}$ & $\mathrm{P} 4_{2} / \mathrm{n}$ \\
\hline $\mathbf{a}(\AA)$ & $10.6852(4)$ & $16.2593(8)$ & $7.2189(5)$ & $18.015(1)$ \\
\hline $\mathbf{b}(\AA)$ & $16.0221(6)$ & $9.4274(4)$ & $19.068(2)$ & $18.015(1)$ \\
\hline c $(\AA)$ & $21.3470(7)$ & $24.049(1)$ & $6.4321(5)$ & $18.438(1)$ \\
\hline $\boldsymbol{\beta}$ (deg.) & $103.969(4)$ & $108.495(5)$ & $107.042(8)$ & 90 \\
\hline $\mathbf{V}\left(\AA^{3}\right)$ & $3546.5(2)$ & $3496.0(3)$ & $846.5(1)$ & $5984.3(6)$ \\
\hline $\mathbf{Z}$ & 2 & 4 & 4 & 8 \\
\hline$\rho_{\text {cal }}\left(\mathrm{g} \cdot \mathrm{cm}^{-3}\right)$ & 1.706 & 1.721 & 2.075 & 1.507 \\
\hline Crystal shape & needle & block & needle & block \\
\hline Crystal color & colorless & colorless & blue & colorless \\
\hline Crystal size $\left(\mathrm{mm}^{3}\right)$ & $0.05 \times 0.07 \times 0.37$ & $0.17 \times 0.23 \times 0.29$ & $0.24 \times 0.26 \times 0.75$ & $0.51 \times 0.64 \times 0.72$ \\
\hline $\boldsymbol{\mu}\left(\mathrm{mm}^{-1}\right)$ & 1.897 & 2.106 & 2.588 & 2.584 \\
\hline $\mathbf{T}(\mathrm{K})$ & 293 & 293 & 293 & 150 \\
\hline No. reflections / $\boldsymbol{R}_{\text {int }}$ & $27103 / 0.040$ & $8721 / 0.032$ & $3966 / 0.024$ & $6785 / 0.034$ \\
\hline No. reflections used & 10608 & 5931 & 1777 & 4836 \\
\hline No. parameters refined & 920 & 531 & 137 & 348 \\
\hline$R(F) / R_{w}(F)[I / \sigma(I)>2]$ & $0.0516 / 0.0664$ & 0.0304 / 0.0333 & $0.0307 / 0.0336$ & $0.0681 / 0.0865$ \\
\hline S & 1.33 & 1.01 & 1.10 & 1.36 \\
\hline$\Delta \rho_{\max } / \Delta \rho_{\min }\left(\mathrm{e}^{-} . \AA^{-3}\right)$ & $+1.91 /-1.84$ & $-0.89 /+1.41$ & $+0.48 /-0.68$ & $+3.28 /-2.17$ \\
\hline Absorption correction & analytical & analytical & analytical & analytical \\
\hline
\end{tabular}


Table S5. Important bond lengths $(\AA \hat{)})$ for complex 1.

\begin{tabular}{|c|c|c|c|c|c|}
\hline Eu1-01 & $2.483(7)$ & Eu1-011 & $2.475(6)$ & Eu1-N9 & $2.533(8)$ \\
\hline Eu1-O21 & $2.452(7)$ & Eu1-N25 & $2.531(7)$ & Eu1-O32 & $2.429(7)$ \\
\hline Eu1-O41 & $2.431(7)$ & Eu1-051 & $2.476(6)$ & Eu1-N49 & $2.536(8)$ \\
\hline Eu38-061 & $2.441(7)$ & Eu38-N65 & $2.543(8)$ & Eu38-071 & $2.486(7)$ \\
\hline Eu38-081 & $2.507(6)$ & Eu38-N89 & $2.522(8)$ & Eu38-091 & $2.418(7)$ \\
\hline Eu38-O141 & $2.432(7)$ & Eu38-N149 & $2.507(8)$ & Eu38-O152 & $2.423(7)$ \\
\hline Mg82-O101 & $2.037(8)$ & Mg82-O102 & $2.084(8)$ & Mg82-O103 & $2.086(9)$ \\
\hline Mg82-O104 & $2.073(7)$ & Mg82-0105 & $2.087(8)$ & Mg82-O106 & $2.046(8)$ \\
\hline Mg75-O111 & $2.086(9)$ & Mg75-0112 & $2.110(9)$ & Mg75-O113 & $2.071(8)$ \\
\hline Mg75-O114 & $2.066(9)$ & Mg75-0115 & $2.090(9)$ & Mg75-O116 & $2.048(9)$ \\
\hline Mg89-0121 & $2.050(9)$ & Mg89-0122 & $2.060(8)$ & Mg89-O123 & $2.101(8)$ \\
\hline Mg89-O124 & $2.069(9)$ & Mg89-0125 & $2.043(9)$ & Mg89-O126 & $2.07(1)$ \\
\hline
\end{tabular}

Table S6. SHAPE ${ }^{14}$ analysis for $\mathrm{Eu}(\mathrm{III})\left\{\mathrm{O}_{8} \mathrm{~N}_{3}\right\}$ environment within crystal structure of 1.

\begin{tabular}{|c|c|c|c|c|c|c|}
\hline \multirow{4}{*}{$\overline{⿱ 亠 䒑}$} & OPY-9 & HBPY-9 & JTC-9 & JCCU-9 & CCU-9 & JCSAPR-9 \\
\hline & 22.87 & 18.49 & 14.17 & 10.03 & 8.80 & 1.98 \\
\hline & CSAPR-9 & JTCTPR-9 & TCTPR-9 & JTDIC-9 & HH-9 & MFF-9 \\
\hline & 1.01 & 2.34 & 1.37 & 10.88 & 11.68 & 1.64 \\
\hline \multirow{4}{*}{ 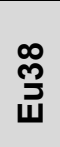 } & OPY-9 & HBPY-9 & JTC-9 & JCCU-9 & CCU-9 & JCSAPR-9 \\
\hline & 23.21 & 17.69 & 13.65 & 9.64 & 8.44 & 2.06 \\
\hline & CSAPR-9 & JTCTPR-9 & TCTPR-9 & JTDIC-9 & HH-9 & MFF-9 \\
\hline & 1.09 & 2.2 & 1.40 & 10.74 & 11.37 & 16 \\
\hline
\end{tabular}

Table S7. Important bond lengths (Á) for complex 2.

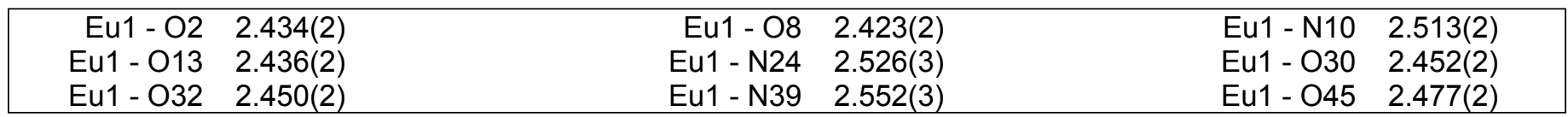

Table S8. SHAPE analysis for $\mathrm{Eu}(\mathrm{III})\left\{\mathrm{O}_{8} \mathrm{~N}_{3}\right\}$ environment within crystal structure of 2.

\begin{tabular}{|c|c|c|c|c|c|c|}
\hline \multirow{4}{*}{$\Xi$} & OPY-9 & HBPY-9 & JTC-9 & JCCU-9 & CCU-9 & JCSAPR-9 \\
\cline { 2 - 7 } & 23.14 & 18.36 & 13.88 & 9.35 & 8.17 & 2.47 \\
\cline { 2 - 7 } & CSAPR-9 & JTCTPR-9 & TCTPR-9 & JTDIC-9 & HH-9 & MFF-9 \\
\cline { 2 - 7 } & 1.57 & 2.07 & 1.14 & 10.35 & 11.96 & 2.15 \\
\hline
\end{tabular}

Table S9. Important bond lengths and distances $(\hat{A})$, bond angles and torsion angles $\left(^{\circ}\right)$ for complex 3.

\begin{tabular}{|c|c|c|c|c|c|}
\hline Cu1-O3 & $2.43242)$ & Cu1-O2 & $1.943(2)$ & Cu1-O3 & $2.062(2)$ \\
\hline Cu1-N7 & $1.899(2)$ & Cu1-O13 & $2.044(2)$ & Cu1-015 & $2.405(2)$ \\
\hline $\begin{array}{l}\mathrm{Cu} \cdots \mathrm{Cu} \cdots \mathrm{Cu} \\
\text { intra-chain }\end{array}$ & 3.869 & $\begin{array}{r}\mathrm{Cu} \cdots \mathrm{Cu} \cdots \mathrm{Cu} \\
\text { Shortest inter-chain }\end{array}$ & 8.222 & & \\
\hline O3-Cu1-O2 & $84.20(7)$ & O3-Cu1-Ö & $99.21(7)$ & O2-Cu1-O3 & $103.74(7)$ \\
\hline O3-Cu1-N7 & $91.98(7)$ & O2-Cu1-N7 & $174.76(7)$ & O3-Cu1-N7 & $80.38(9)$ \\
\hline O3-Cu1-013 & $83.59(8)$ & O2-Cu1-O13 & $95.61(8)$ & O3-Cu1-013 & $160.62(6)$ \\
\hline N7-Cu1-O13 & $80.37(9)$ & O3-Cu1-015 & $169.56(6)$ & O2-Cu1-015 & $88.28(7)$ \\
\hline
\end{tabular}


Table S10. Important bond lengths $(\AA \hat{)})$ for complex 4.



Table S11. SHAPE analysis for $\mathrm{Tb}(\mathrm{III})\left\{\mathrm{O}_{4} \mathrm{~N}_{5}\right\}$ environment within crystal structure of 4 .

\begin{tabular}{|c|c|c|c|c|c|c|}
\hline \multirow{4}{*}{$\frac{5}{1}$} & OPY-9 & HBPY-9 & JTC-9 & JCCU-9 & CCU-9 & JCSAPR-9 \\
\hline & 23.08622 & 17.38200 & 15.20241 & 8.03853 & 6.98477 & 2.75143 \\
\hline & CSAPR-9 & JTCTPR-9 & TCTPR-9 & JTDIC-9 & $\mathrm{HH}-9$ & \\
\hline & 1.80723 & 2.90731 & 2.18716 & 11.87311 & 7.71756 & \\
\hline
\end{tabular}




\section{6) Photophysical measurements in solution.}

Emission spectra were measured using Horiba-Jobin-Yvon Fluorolog-3 fluorimeter on concentrated solutions $\left(10^{-3} \mathrm{M}\right)$. The steady-state luminescence was excited by unpolarized light from a $450 \mathrm{~W}$ xenon continuous wave (CW) lamp and detected at an angle of $22.5^{\circ}$ for measurements of dilute solutions (10 mm quartz cuvette) either by using a Hamamatsu R928 or Peltier cooled R2658 photomultiplier tube. Spectra were corrected for both excitation source light-intensity variation and emission spectral responses.

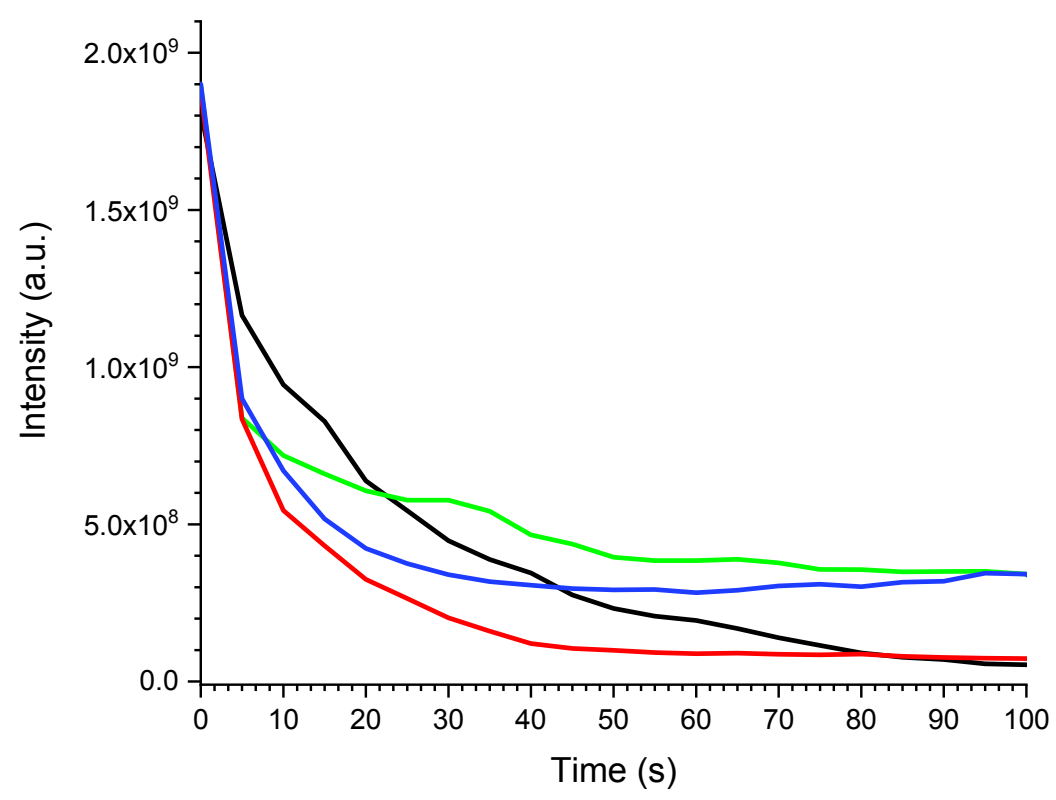

Figure S5. Evolution of the Eu(III) emission intensity after addition of 1 equivalent of divalent transition metal to $[\mathrm{Na}]_{3}\left[\mathrm{Eu}(\mathrm{DPA})_{3}\right]$ at time $=0 \mathrm{~s}\left(\mathrm{C}\left([\mathrm{Na}]_{3}\left[\mathrm{Eu}(\mathrm{DPA})_{3}\right)=\mathrm{C}\left(\mathrm{MCl}_{2}\right)=1,0610^{-3} \mathrm{M}\right.\right.$, $\left[\mathrm{MCl}_{2}\right]=\mathrm{CdCl}_{2}$ (green), $\mathrm{ZnCl}_{2}$ (blue), $\mathrm{CoCl}_{2}$ (red) or $\mathrm{NiCl}_{2}$ (black), $\lambda_{\mathrm{exc}}=283 \mathrm{~nm}, \lambda_{\mathrm{em}}=614 \mathrm{~nm}$, front face, $\mathrm{H}_{2} \mathrm{O}$, room temperature). 


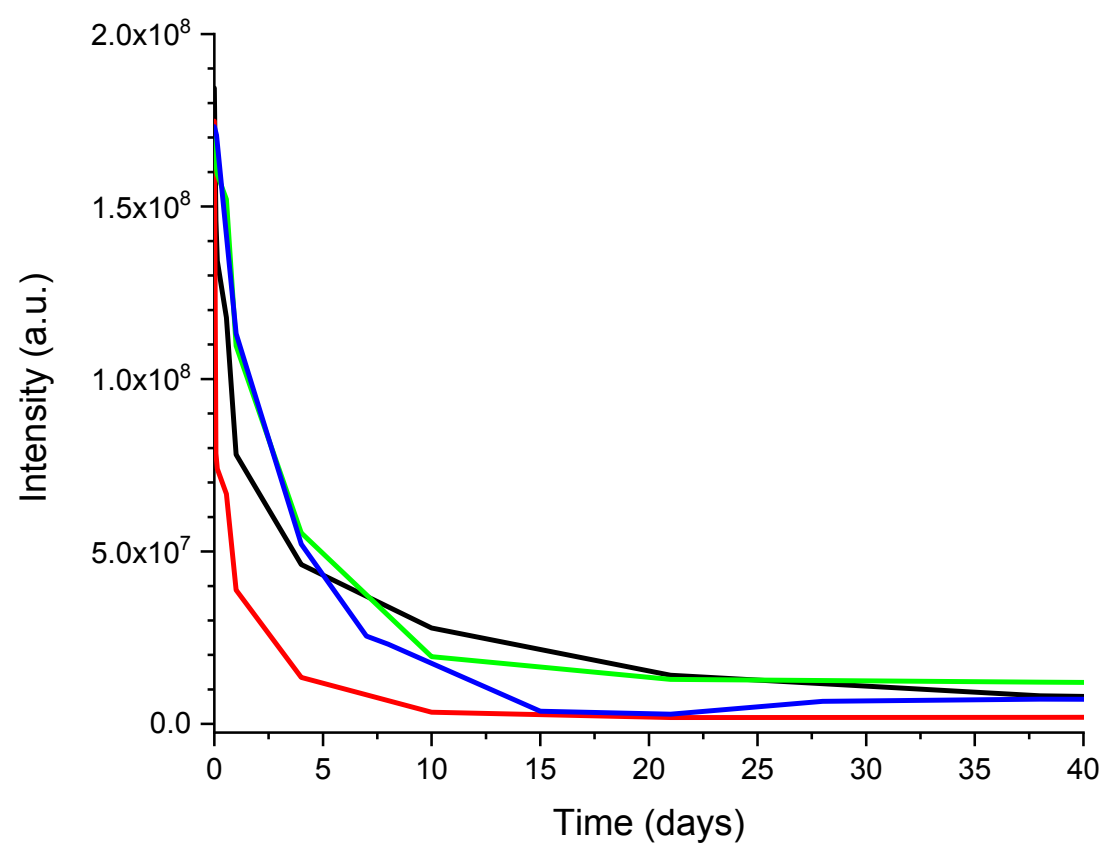

Figure S6. Evolution of the $\mathrm{Tb}$ (III) emission intensity of Tb-Xo4 after addition of 1 equivalent of divalent transition metal $\left(\mathrm{C}[\mathrm{Tb}-\mathrm{Xo} 4]=\mathrm{C}\left[\mathrm{MCl}_{2}\right] \sim 10^{-3} \mathrm{M},\left[\mathrm{MCl}_{2}\right]=\mathrm{CdCl}_{2}\right.$ (green), $\mathrm{ZnCl}_{2}$ (blue), $\mathrm{CoCl}_{2}$ (red) or $\mathrm{NiCl}_{2}$ (black), $\lambda_{\mathrm{exc}}=273 \mathrm{~nm}, \lambda_{\mathrm{em}}=488 \mathrm{~nm}$, front face, $\mathrm{H}_{2} \mathrm{O}$, room temperature)

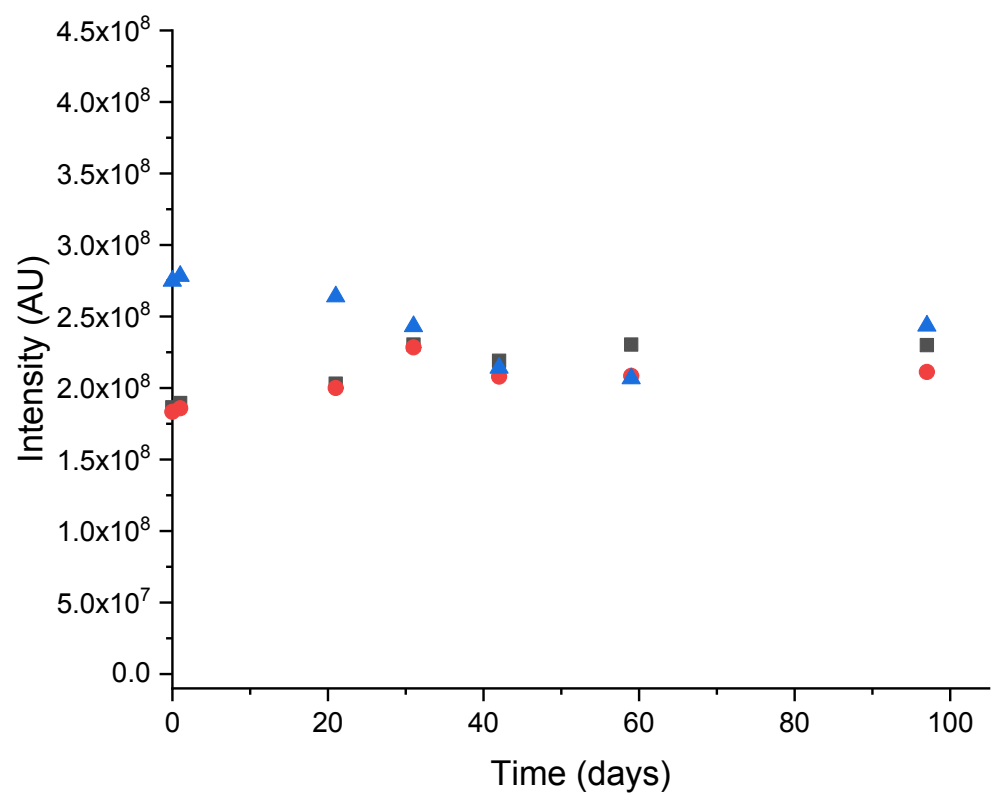

Figure S7. Evolution of the Tb-Xo4 emission intensity after addition of 1-10 eq of alkaline-earth salts $\left(\mathrm{C}[\mathrm{Tb}-\mathrm{Xo} 4] \sim 1 \mathrm{mM}, \mathrm{C}\left[\mathrm{CaCl}_{2}\right]=1.1\right.$ to $11 \mathrm{mM}(\boldsymbol{\square}), \mathrm{C}\left[\mathrm{MgCl}_{2}\right]=1.1$ to $11 \mathrm{mM}(\mathrm{O}), \mathrm{C}\left[\mathrm{BaAc}_{2}\right]$ $=1.0$ to $10 \mathrm{mM}(\Delta), \lambda_{\mathrm{exc}}=273 \mathrm{~nm}, \lambda_{\mathrm{em}}=488 \mathrm{~nm}$, front face, $\mathrm{H}_{2} \mathrm{O}$, room temperature). 


\section{References and notes.}

1 Tancrez, N. ; Feuvrie, C. ; Ledoux, I. ; Zyss, J. ; Toupet, L. ; Le Bozec, H. ; Maury O., Lanthanide Complexes for Second Order Nonlinear Optics: Evidence for the Direct Contribution of f Electrons to the Quadratic Hyperpolarizability. J. Am. Chem. Soc., 2005, 127, 13474-13475.

2 Engilberge, S., Riobé, F., Di Pietro, S. ; Lassalle, L. ; Coquelle, N. ; Arnaud, C. ; Madern, D. ; Breyton, C. ; Maury, O. ; Girard E., Crystallophore: a versatile lanthanide complex for protein crystallography combining nucleating effects, phasing properties, and luminescence Chem. Science. 2017, 8, 5909-5917.

3 Jiang, T.; Roux, A. ; Engilberge, S. ; Alsalman, Z. ; Di Pietro, S. ; Franzetti, B. ; Riobé, F. ; Maury, O. ; Girard E., Tracking Crystallophore Nucleating Properties: Setting Up a Database for Statistical Analysis. Cryst. Growth \& Design. 2020, 20, 5322-5329

4 a) Pos, K.M.; Diederichs, K., Purification, Crystallization and Preliminary Diffraction Studies of AcrB, an Inner-Membrane Multi-Drug Efflux Protein. Acta Crystallogr., D Biol. Crystallogr. 2002, 58,1865-1867 ; b) Stroebel D. ; Sendra, V. ; Cannella, D. ; Helbig, K. ; Covès J., Oligomeric Behavior of the RND Transporters CusA and AcrB in Micellar Solution of Detergent. Biochimica et Biophysica Acta 2007, 1768, 1567-1573.

5 a) Wagner, T., Koch, J., Ermler, U., Shima, S., Methanogenic Heterodisulfide Reductase (HdrABC-MvhAGD) uses two Noncubane [4Fe-4S] Clusters for Reduction. Science, 2017, 357, 699-703.; b) Engilberge, S.; Wagner, T.; Santoni, G.; Breyton, C.; Shima, S.; Franzetti, B.; Riobe, F.; Maury, O.; Girard, E. Protein Crystal Structure Determination with the Crystallophore, a Nucleating and Phasing agent. J. Appl. Crystallogr. 2019, 52, 722.

6 Engilberge, S.; Riobé, F.; Wagner, T.; Di Pietro, S.; Breyton, C.; Franzetti, B.; Shima, S.; Girard, E.; Dumont, E.; Maury O., Unveiling the Binding Modes of the Crystallophore, a Terbium-based Nucleating and Phasing Molecular Agent for Protein Crystallography. Chem. Eur. J. 2018, 24, 9739-9746.

7 Søndergaard, C. R.; Olsson, M. H. M.; Rostkowski, M.; Jensen J. H. Improved Treatment of Ligands and Coupling Effects in Empirical Calculation and Rationalization of pKa Values. J. Chem. Theory Comput. 2011, 7, 2284-2295

8 Gaussian 16, Revision B.01, Frisch M. J.; Trucks, G. W.; Schlegel, H. B.; Scuseria, G. E.; Robb, M. A.; Cheeseman, J. R.; Scalmani, G.; Barone, V.; Petersson, G. A.; Nakatsuji, H.; Li, X.; Caricato, M.; Marenich, A. V.; Bloino, J.; Janesko, B. G.; Gomperts, R.; Mennucci, B.; Hratchian, H. P.; Ortiz, J. V.; Izmaylov, A. F.; Sonnenberg, J. L.; Williams-Young, D.; Ding, F.; Lipparini, F.; Egidi, F.; Goings, J.; Peng, B.; Petrone, A.; Henderson, T.; Ranasinghe, D.; Zakrzewski, V. G.; Gao, J.; Rega, N.; Zheng, G.; Liang, W.; Hada, M.; Ehara, M.; Toyota, K.; Fukuda, R.; Hasegawa, J.; Ishida, M.; Nakajima, T.; Honda, Y.; Kitao, O.; Nakai, H.; Vreven, 
T.; Throssell, K.; Montgomery, J. A., Jr.; Peralta, J. E.; Ogliaro, F.; Bearpark, M. J.; Heyd, J. J.; Brothers, E. N.; Kudin, K. N.; Staroverov, V. N.; Keith, T. A.; Kobayashi, R.; Normand, J.; Raghavachari, K.; Rendell, A. P.; Burant, J. C.; Iyengar, S. S.; Tomasi, J.; Cossi, M.; Millam, J. M.; Klene, M.; Adamo, C.; Cammi, R.; Ochterski, J. W.; Martin, R. L.; Morokuma, K.; Farkas, O.; Foresman, J. B.; Fox, D. J. Gaussian, Inc., Wallingford CT, 2016.

9 CrysAlisPro, v. 1.171.33.46 (rel. 27-08-2009 CrysAlis171.NET), Oxford Diffraction Ltd., 2009.

10 De Meulenaer, J.; Tompa, H., The Absorption Correction in Crystal Structure Analysis. Acta Crystallogr. 1965, 19, 1014-1018.

11 Blessing, R. H., An Empirical Correction for Absorption Anisotropy Acta Crystallogr., Sect. A: Found. Crystallogr. 1995, 51, 33-38.

12 Altomare, A. ; Burla, M. C. ; Camalli, M. ; Cascarano, G. L. ; Giacovazzo, C. ; Guagliardi, A. ; Moliterni, A. G. G. ; Polidori, G. ; Spagna, R. SIR97: a New Tool for Crystal Structure Determination and Refinement. J. Appl. Crystallogr. 1999, 32, 115-119.

13 Watkin, D. J.; Prout, C. K.; Carruthers, J. R.; Betteridge P. W. in CRISTAL Issue 11, Chemical Crystallography Laboratory, Oxford, 1999.

14 Llunell, M.; Casanova, D.; Cirera, J.; Bofill, J. M.; Alemany, P.; Alvarez, S. SHAPE (Version 2.1) 2013 